DRAFT VERSION MARCH 12, 2018

Preprint typeset using $\mathrm{LT}_{\mathrm{E}} \mathrm{X}$ style emulateapj v. 4/12/04

\title{
ALMA SUBMILLIMETER CONTINUUM IMAGING OF THE HOST GALAXIES OF GRB 021004 AND GRB 080607
}

\author{
Wei-HaO WANG ${ }^{1}$, HSIAO-WEN CHEN $^{2}$, AND Kui-Yun HuAng ${ }^{1}$ \\ Draft version March 12, 2018
}

\begin{abstract}
We report $345 \mathrm{GHz}$ continuum observations of the host galaxies of gamma-ray bursts (GRBs) 021004 and 080607 at $z>2$ using the Atacama Large Millimeter/Submillimeter Array (ALMA) in Cycle 0. Of the two bursts, GRB 021004 is one of the few GRBs that originates in a Lyman limit host, while GRB 080607 is classified as a "dark burst" and its host galaxy is a candidate of dusty star forming galaxy at $z \sim 3$. With an order of magnitude improvement in the sensitivities of the new imaging searches, we detect the host galaxy of GRB 080607 with a flux of $S_{345}=0.31 \pm 0.09 \mathrm{mJy}$ and a corresponding infrared luminosity of $L_{\mathrm{IR}}=(2.4-$ $4.5) \times 10^{11} L_{\odot}$. However, the host galaxy of GRB 021004 remains undetected and the ALMA observations allow us to place a 3- $\sigma$ upper limit of $L_{\mathrm{IR}}<3.1 \times 10^{11} L_{\odot}$ for the host galaxy. The continuum imaging observations show that the two galaxies are not ultraluminous infrared galaxies but are at the faintest end of the dusty galaxy population that gives rise to the submillimeter extragalactic background light. The derived star formation rates of the two GRB host galaxies are less than $100 M_{\odot} \mathrm{yr}^{-1}$, which are broadly consistent with optical measurements. The result suggests that the large extinction $\left(A_{V} \sim 3\right)$ in the afterglow of GRB 080607 is confined along its particularly dusty sightline, and not representative of the global properties of the host galaxy. Subject headings: galaxies: high-redshift — submillimeter: galaxies — Gamma-ray burst: individual (021004, 080607)
\end{abstract}

\section{INTRODUCTION}

Long-duration gamma-ray bursts (GRBs) are believed to originate in the death of massive stars (see Woosley \& Heger 2006 for a recent review), and are thus expected to trace star formation in galaxies (e.g., Wijers et al. 1998; Totani 1999). Because of the extreme luminosity of the prompt emission and the afterglow, GRBs are a powerful probe of star formation in early times (e.g., Tanvir et al. 2009; Salvaterra et al. 2009). In order to establish the link between GRBs and the cosmic star formation, it is important to understand the properties of the GRB host galaxies (Hjorth et al. 2012). A critical measurement is the star formation rates (SFRs) of the host galaxies.

There exist various SFR indicators for high-redshift galaxies in different spectral windows. One key concern is the effect of dust extinction. Even with arguably good extinction corrections in optical data, highly obscured components may still exist and would only appear at the far-infrared and radio wavelengths. The presence of such components would indicate a significant spatial variation in dust content in which case a global extinction correction would not apply. For GRB host galaxies, systematic surveys were carried out to observe continuum emission in the radio (Michałowski et al. 2012) and submillimeter (Berger et al. 2003; Tanvir et al. 2004; Priddey et al. 2006) frequency ranges for constraining dust enshrouded SFR. Because of the synchrotron spectral slope, radio observations are only effective in detecting GRB host galaxies at $z \lesssim 1$ (e.g., Michałowski et al. 2012). Dust continuum emission in the submillimeter has a spectral slope that can nearly cancel the effect of luminosity distance from $z \sim 1$ to $z \sim 10$, making the submillimeter wavelengths an effective window for detecting faint galaxies at high redshifts (Blain \& Longair 1993). However, the $850 \mu \mathrm{m}$ survey of $21 \mathrm{GRB}$ host galaxies at $z<3.5$ by Tanvir et al. (2004) using the James

\footnotetext{
1 Academia Sinica Institute of Astronomy and Astrophysics, P.O. Box 23141, Taipei 10617, Taiwan

${ }^{2}$ Department of Astronomy \& Astrophysics and Kavli Institute for Cosmological Physics, University of Chicago, Chicago, IL 60637, USA
}

Clerk Maxwell Telescope (JCMT) only uncovered three host galaxies at $>3 \sigma$ confidence levels, and all three hosts are at $z<1.5$. Submillimeter single-dish telescopes are confusion limited at roughly a few mJy at $850 \mu \mathrm{m}$, and therefore can only detect galaxies with infrared luminosities of $L_{\mathrm{IR}}$ (8 to $1000 \mu \mathrm{m})>10^{12.5} L_{\odot}$, or SFR $\gtrsim 1000 M_{\odot} \mathrm{yr}^{-1}$. This SFR limit is much larger than the typical SFR of GRB host galaxies measured in the optical (Christensen, Hjorth, \& Gorosabel 2004; Savaglio, Glazebrook, \& Le Borgne 2009). Deeper submillimeter measurements are thus required to better constrain their infrared luminosity and the underlying SFR.

A particularly interesting class of GRB is "dark GRBs" (Djorgovski et al. 2001; Jakobsson et al. 2005), defined by their faint optical afterglow, relative to the bright X-ray emission. A definition for dark bursts is those with the opticalto-X-ray spectral index of $\beta_{\mathrm{OX}}<0.5$ (Jakobsson et al. 2004), which is physically motivated based on theoretical predictions of the synchrotron model. Approximately 30\%-50\% of longduration GRBs have suppressed optical fluxes relative to their X-ray emission (Melandri et al. 2009; Cenko et al. 2009; Melandri et al. 2012). The weaker optical emission can be caused by either intergalactic medium absorption at $z>6$ (Kawai et al. 2006; Greiner et al. 2009; Tanvir et al. 2009; Salvaterra et al. 2009) or dust extinction in their host galaxies (Perley et al. 2009). In the latter case, dark GRBs may serve as a tracer of dust enshrouded star formation across cosmic time. However, uncertainty remains regarding whether the observed dust obscuration is representative of the global properties of the host galaxies or merely local to the progenitor site. This uncertainty can be addressed by comparing the rest-frame infrared luminosities between dark GRB host galaxies and the rest of the host galaxy population.

In this letter, we present initial results from a pilot study of GRB host galaxies in the submillimeter frequency range using the Atacama Large Millimeter/Submillimeter Array (ALMA). In Cycle 0, we observed the host galaxies of GRB 021004 $(z=2.330)$ and GRB $080607(z=3.036)$ at $345 \mathrm{GHz}$. The GRB fields were selected to have early-time afterglow spectra 
available for constraining the ISM absorption properties of the host galaxies (e.g., Fynbo et al. 2005; Prochaska et al. 2009; Sheffer et al. 2009). They are among the best studied events and they represent two extremes in the integrated total ISM column density alone the afterglow line of sight. GRB 021004 is one of a few GRBs arising in a Lyman limit absorber with $N_{\mathrm{HI}}=10^{19.5 \pm 0.5} \mathrm{~cm}^{-2}$, and the host of GRB 080607 is a damped Ly $\alpha$ absorber with an unprecedentedly high gas den-

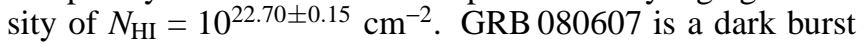
with highly extinguished afterglow $\left(A_{V} \sim 3.2\right.$, Prochaska et al. 2009; Perley et al. 2011), and the extinction suggests that the host galaxy may have detectable submillimeter dust emission. The host galaxies of the two GRBs are found to have optical SFRs of 10-40 $M_{\odot} \mathrm{yr}^{-1}$ (Jakobsson et al. 2005; Castro-Tirado et al. 2010; Chen, Perley, \& Wilson et al. 2011), consistent with normal star forming galaxies at $z>2$. Here we use the ALMA results to estimate the infrared luminosities of the two host galaxies, and to examine whether there exist highly obscured star-forming regions that are not revealed by optical observations. We describe our ALMA observations and data reduction in Section 2, and the results and estimate the infrared luminosities and the SFRs of the GRB host galaxies in Section 3. The implication of our observations is discussed in Section 4. We adopt cosmological parameters of $H_{0}=71$ $\mathrm{km} \mathrm{s}^{-1} \mathrm{Mpc}^{-1}, \Omega_{M}=0.27$, and $\Omega_{\Lambda}=0.73$, and we convert the previous results to this set of cosmology.

\section{OBSERVATION AND DATA REDUCTION}

Observations of the continuum emission at $345 \mathrm{GHz}$ from the host galaxies of GRB 021004 and GRB 080607 ware obtained using the ALMA 12-m array. Four spectral windows were tuned to center at 338, 340,350, and $352 \mathrm{GHz}$, each with a $2 \mathrm{GHz}$ bandwidth. Bandpass calibrators and flux calibrators were observed prior to the observations of the science targets. Bright quasars near the GRB fields were observed every $\sim 11$ minutes for phase and amplitude calibrations. For each science target, a total of $0.7-0.8 \mathrm{hr}$ of on-target integration was collected. Table 1 summarizes the basic observing parameters and the various calibrators.

We received the data from the Joint ALMA Observatory (JAO) a few weeks after the observations. The delivered data were already bandpass, flux, and gain (phase and amplitude) calibrated by JAO, and reference images were also provided. All the above calibration and imaging were carried out using Common Astronomy Software Applications (CASA, McMullin et al. 2007). We further inspected the JAO calibration in CASA, and Fourier-transformed the complex visibility to make our own images. To obtain the highest $\mathrm{S} / \mathrm{N}$, we gave all visibility data equal weights regardless of their density distribution in the $u v$ plane (i.e., "natural weighting"). The resulting synthesized beams and sensitivities are summarized in Table 1. We do not detect the host galaxy of GRB 021004, and thus the imaging remains in the "dirty" stage. We detect the host galaxy of GRB 080607, and therefore "CLEANed" the sidelobes of the detected object in CASA.

\section{RESULTS}

\subsection{GRB 021004}

We do not detect the host galaxy of GRB 021004 (Figure 1). The $345 \mathrm{GHz}$ point-source flux measured at the location of the host galaxy is $0.17 \pm 0.11 \mathrm{mJy}$. Tanvir et al. (2004) measured an $850 \mu \mathrm{m}$ flux of $0.77 \pm 1.25 \mathrm{mJy}$ using SCUBA on JCMT. Smith et al. (2005) improved the previous SCUBA result slightly to $-1.4 \pm 1.0 \mathrm{mJy}$. Our measurement is $10 \times$
TABLE 1

OBSERVING LOG

\begin{tabular}{lcc}
\hline \hline \multicolumn{1}{c}{ Target } & GRB 021004 & GRB 080607 \\
\hline Observing Date & Oct 22, 2011 & Nov 16, 2011 \\
& Nov 5, 2011 & Jan 12, 2012 \\
Number of Antennas $^{\mathrm{a}}$ & 17 & 20 \\
On-Target Integration & $42.6 \mathrm{~min}$ & $48.2 \mathrm{~min}$ \\
Bandpass Calibrator & $3 \mathrm{c} 454.3$ & $3 \mathrm{c} 273$ \\
Flux Calibrator & Callisto & Titan \\
Gain Calibrators & B0007+016 & $\mathrm{J} 1239+075$ \\
& J0010+109 & B1236+077 \\
Sensitivity (1 $\sigma$ ) & $0.113 \mathrm{mJy}$ & $0.098 \mathrm{mJy}$ \\
Synthesized Beam & $1^{\prime \prime} 55 \times 1$ I. $^{\prime} 25,-20^{\circ}$ & $1^{\prime \prime} 56 \times 00^{\prime \prime} 87,3^{\circ}$ \\
(major $\times$ minor, PA) & & \\
\hline
\end{tabular}

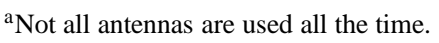

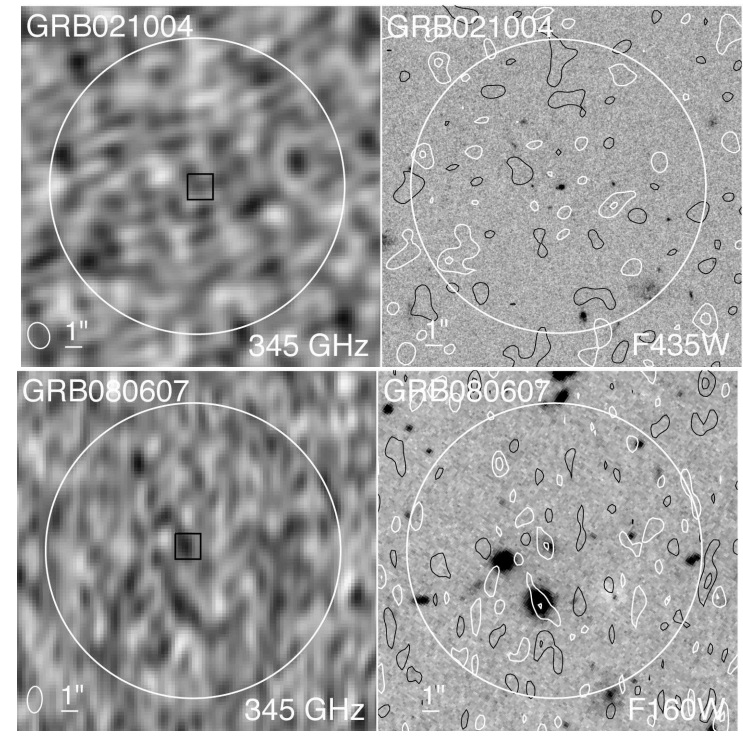

FIG. 1.- ALMA (left) and HST (right) images of the GRB host galaxies. In all panels, the images have inverted brightness scales. The large circles indicate the FWHM of the ALMA primary beam, which is 17!" 4. In the ALMA $345 \mathrm{GHz}$ panels, black boxes of 1"' 5 sizes indicate the optical positions of the host galaxies, and ellipses in the lower left indicate the synthesized beams. In the HST panels (ACS F435W for GRB 021004 and WFC3 F160W for GRB 080607), contours of $345 \mathrm{GHz}$ flux are overlaid. White contours are +1.5 and $+3.0 \sigma$ and black contours are -1.5 and $-3.0 \sigma$, where $1 \sigma$ is 0.113 and $0.098 \mathrm{mJy}^{\text {beam }}{ }^{-1}$ for GRB 021004 and GRB 080607, respectively.

deeper than these previous observations but the host galaxy remains undetected.

Given the redshift of $z=2.330$ and adopting the 3- $\sigma$ upper limit of $0.33 \mathrm{mJy}$, we can estimate the upper limits of its rest-frame infrared luminosity and SFR. To do so with single-band photometry, we need to assume a dust temperature, and this can be done using the infrared spectral energy distribution (SED) library of Chary \& Elbaz (2001, hereafer CE01). The SEDs in CE01 are luminosity dependent (based on a locally calibrated luminosity - dust temperature relation) and do not allow for scaling of the SEDs. The library contains a broad range of infrared luminosity, from $2 \times 10^{8}$ to $4 \times 10^{13} L_{\odot}$, and each template has its unique dust temperature. We thus redshift the CE01 SEDs to $z=2.330$ and look for those with observed $345 \mathrm{GHz}$ fluxes below our upper limit. Of the 105 templates provided by CE01, 65 have $345 \mathrm{GHz}$ fluxes lower than $0.33 \mathrm{mJy}$ (Figure 2), with corresponding infrared luminosities between $2.6 \times 10^{8}$ and $3.1 \times 10^{11} L_{\odot}$. 

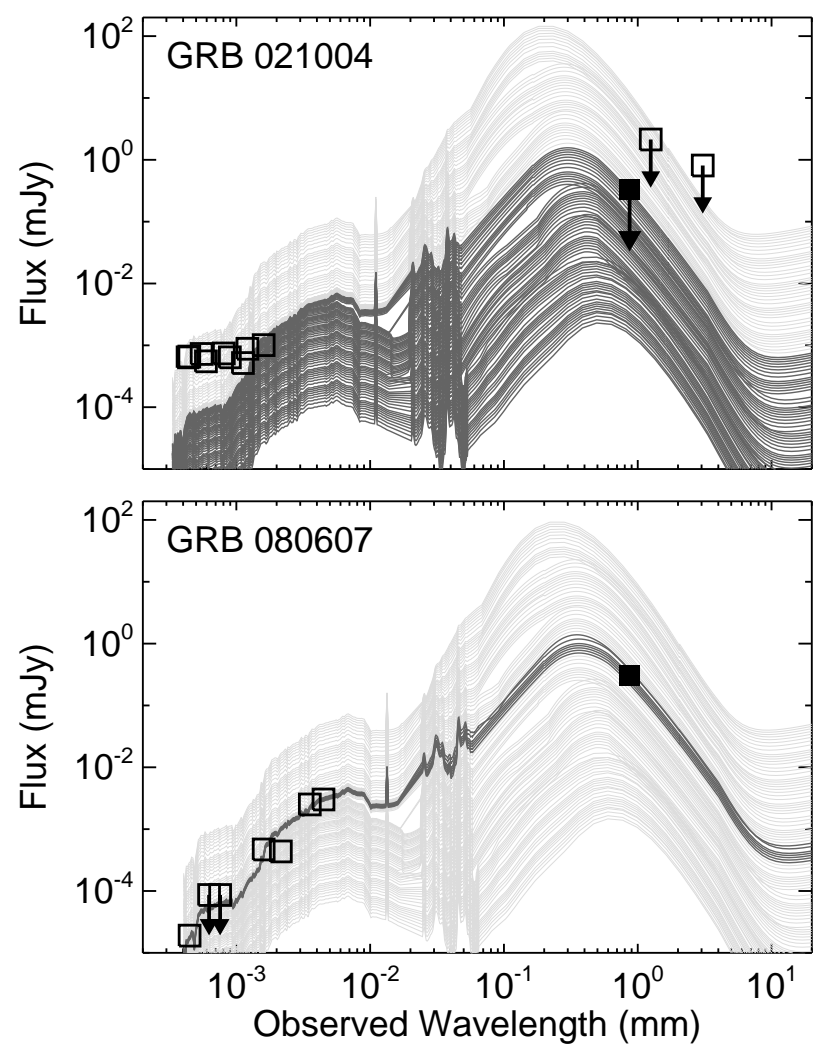

FIG. 2.- ALMA constraints (solid squares) on the infrared SEDs of the GRB hosts. The curves are the series of 105 redshifted CE01 SED templates, which have infrared luminosities from $2 \times 10^{8}$ to $4 \times 10^{13} L_{\odot}$. The thick curves are the CE01 templates that satisfy our ALMA measurements. We also show the multiwavelength photometry from the literature (open squares; GRB 021004: Fynbo et al. 2005; de Ugarte Postigo et al. 2005; GRB 080607: Chen et al. 2010; Chen, Perley, \& Wilson et al. 2011) but we do not use them in the SED fitting. Error bars are all smaller than the symbols and all upper limits are $3 \sigma$.

An ultraluminous infrared galaxy (ULIRG, $L_{\mathrm{IR}}>10^{12} L_{\odot}$ ) is clearly ruled out for the host of GRB 021004, which can be at most a modest infrared luminous galaxy of $L_{\mathrm{IR}} \sim 3 \times 10^{11}$ $L_{\odot}$. Combining available optical photometric measurements (open squares in Figure 2) with the ALMA upper limit further reveals a blue SED that is inconsistent with any of the CE01 templates, suggesting that the host of GRB 021004 contains primarily young stars with little dust (e.g., Chen et al. 2009) and that the infrared luminosity may be substantially lower than the observed limit. If we adopt the SFR conversion of star-forming galaxies, SFR $\left(M_{\odot} \mathrm{yr}^{-1}\right)=1.7 \times 10^{-10} L_{\mathrm{IR}} / L_{\odot}$ (Kennicutt 1998), then the 3- $\sigma$ upper limit of the SFR of the host galaxy is $53 M_{\odot} \mathrm{yr}^{-1}$. These results are summarized in Table 2.

Castro-Tirado et al. (2010) obtained an optical spectrum of the early-time afterglow of GRB 021004 . The authors estimated an unobscured SFR of $\sim 40 M_{\odot} \mathrm{yr}^{-1}$ based on the observed $\mathrm{H} \alpha$ flux. Jakobsson et al. (2005) obtained a Ly $\alpha$ spectrum of the host galaxy and estimated an SFR of $\sim 11$ $M_{\odot} \mathrm{yr}^{-1}$, but this is uncertain because of the complex radiative transfer of Ly $\alpha$. Both results are within our 3- $\sigma$ upper limit. The ALMA imaging observation confirms the low SFR of the host galaxy, and also shows that more sensitive, multiwavelength ALMA submillimeter imaging is needed to constrain the infrared luminosity and dust SED of this object.

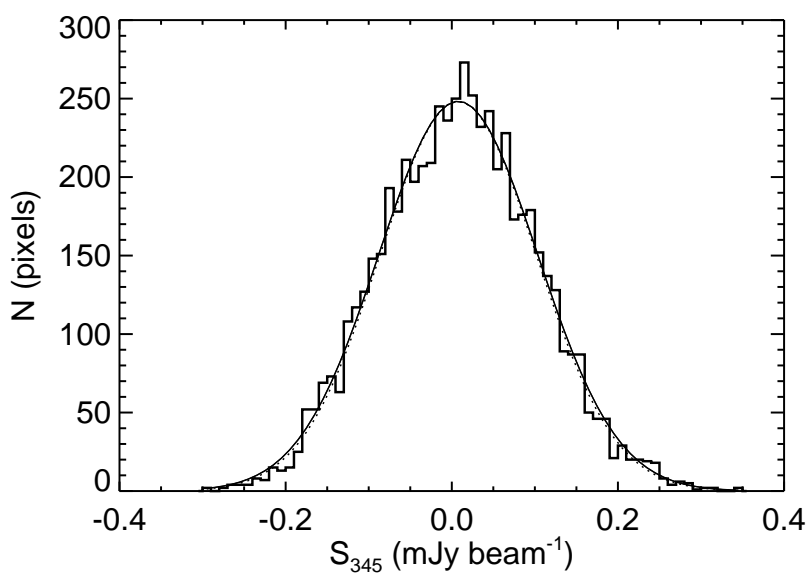

FIG. 3.- Flux distribution of the GRB 080607 ALMA image within the primary beam. The histogram is fitted with a Gaussian with $\sigma=0.096$ mJy beam ${ }^{-1}$ (solid curve), consistent with our measured noise of $0.094 \mathrm{mJy}$ beam $^{-1}$ (dotted curve).

\subsection{GRB 080607}

A significant $345 \mathrm{GHz}$ flux is detected at the location of GRB 080607. Figure 1 shows our ALMA image of the field around GRB 080607 , and its $345 \mathrm{GHz}$ flux contours overlaid on an HST WFC3 F160W image. The rms noise is measured to be $0.094 \mathrm{mJy}^{\text {beam }}{ }^{-1}$ within the primary beam. There exists a peak of $345 \mathrm{GHz}$ emission at the location of the host galaxy, with a peak flux of $0.27 \mathrm{mJy}^{\text {beam }}{ }^{-1}$. We measure a flux of $0.31 \mathrm{mJy}$ by fitting the emission with a point-source model. On the other hand, the contours in Figure 1 suggest that the emission is elongated, and the elongation is similar to that observed in the HST image. We therefore also fit the emission with an extended 2-D Gaussian, and obtain a slightly higher integrated flux of $0.32 \mathrm{mJy}$. However, the fitted Gaussian is still consistent with a point source, which is not surprising given the low $\mathrm{S} / \mathrm{N}$. In the subsequent analyses, we adopt the more conservative measurement of $0.31 \mathrm{mJy}$ with a statistical significance of $3.3 \sigma$. Approximately 3".5 south of the GRB host galaxy, a marginal $(\sim 3 \sigma)$ submillimeter emission is also detected at an optically bright $\mathrm{Mg}$ II absorber at $z=1.3399$ (H.-W. Chen et al. 2012, in preparation). We do not give further consideration to this object in this paper, but we note that this Mg II absorber could also be a faint submillimeter source.

The key question here is whether we can consider the $\sim 3.3 \sigma$ emission as a detection of the GRB host galaxy. First, the fitted $345 \mathrm{GHz}$ peak in both the point-source and the Gaussian cases has an offset of 0 "' 08 from the centroid of the optical emission. This offset is negligible given the $\mathrm{S} / \mathrm{N}$ and the synthesized beam size of 1 ! $^{\prime \prime} 5 \times 0.0^{\prime \prime} 87$. Thus the confidence of the detection is enhanced by its coincident position with the GRB host galaxy.

Second, we consider the probability for this peak to be spurious. Figure 3 shows the histogram of pixel brightness in the primary beam. The distribution can be well fitted with a Gaussian with $\sigma=0.096 \mathrm{mJy}^{\text {beam }}{ }^{-1}$ (solid curve), consistent with our measured noise of $0.094 \mathrm{mJy}^{\text {beam }}{ }^{-1}$ (dotted curve). Following the Gaussian distribution function, the probability of finding a $>3.3 \sigma$ noise spike is $5 \times 10^{-4}$. However, there are hints of a non-Gaussian noise. The histogram in Figure 3 suggests an excess of positive pixels (at $>0.2 \mathrm{mJy}$ ). In the image (Figure 1), additional to the GRB host galaxy, there is a 
TABLE 2

Properties of THE GRB Host Galaxies

\begin{tabular}{ccccc}
\hline \hline Target & Redshift & $S_{345}(\mathrm{mJy})$ & $L_{\mathrm{IR}}\left(10^{11} L_{\odot}\right)$ & $\mathrm{SFR}\left(M_{\odot} \mathrm{yr}^{-1}\right)$ \\
\hline GRB 021004 $^{\mathrm{a}}$ & 2.330 & $<0.33$ & $<3.1$ & $<53$ \\
GRB 080607 & 3.036 & $0.31 \pm 0.09$ & $2.4-4.5$ & $41-77$ \\
\hline
\end{tabular}

${ }^{\text {a }}$ The upper limits for GRB 021004 are all $3 \sigma$.

second $>3.3 \sigma$ spike to the north-east of the GRB host, which has no known optical counterpart. Within the ALMA primary beam $(\mathrm{FWHM}=17$. ! 4$)$, there are approximately 220 independent resolution elements. This additional $>3.3 \sigma$ spike suggests a probability of $1 / 220=5 \times 10^{-3}$, which is $10 \times$ higher than the Gaussian probability. This is an upper limit, since we cannot rule out this spike as a real submillimeter source. Thus the probability of finding a $>3.3 \sigma$ spike at the location of our target is between $5 \times 10^{-4}$ (assuming a Gaussian noise) and $5 \times 10^{-3}$ (assuming that the second $3.3 \sigma$ spike is due to noise). Both these values are sufficiently small. Therefore, the fact that the observed emission coincides with the position of the GRB host substantially increases the confidence level of the detection of the host. In this paper, we consider this a detection, but we also point out that it will be worthwhile to confirm this with ALMA in future larger GRB host galaxy surveys.

With the above measured flux and the redshift of $z=3.036$, we then estimate the infrared luminosity of the host galaxy of GRB 080607, using the CE01 library. We redshift the CE01 templates to $z=3.036$ and find seven of the 105 templates fall in the observed range of $0.313 \pm 0.094 \mathrm{mJy}$ (Figure 2), with $L_{\mathrm{IR}}=2.4 \times 10^{11}$ to $4.5 \times 10^{11} L_{\odot}$. Including available optical and near-infrared photometric measurements, the bottom panel of Figure 2 further shows that the SED is well represented by known dusty templates of local galaxies across the full spectral range. The agreement strongly supports the conclusion that the host galaxy of GRB 080607 is similar to a luminous infrared galaxy (LIRG, $L>10^{11} L_{\odot}$ ) in the local universe. The inferred SFR is between 41 and $77 M_{\odot} \mathrm{yr}^{-1}$. The above results are summarized in Table 2.

Chen, Perley, \& Wilson et al. (2011) presented SED fitting for the host of GRB 080607 at $\lambda_{\text {rest }} \sim 0.4-4 \mu \mathrm{m}$. Adopting a Milky-Way type dust extinction law, they found $A_{V}=1.24$ and an extinction corrected SFR of $\sim 16-24 M_{\odot} \mathrm{yr}^{-1}$, roughly $3 \times$ lower than the submillimeter SFR. Given known uncertainties in both the optical extinction correction and the infrared SED of galaxies and possible variation in the distribution of dust content, the factor of three difference between the optical and submillimeter SFRs only suggests a modest amount of dust enshrouded star formation.

\section{DISCUSSION}

With the pilot ALMA imaging program of two GRB host galaxies at $z>2$, we attempt to constrain the far-infrared properties of GRB host galaxies. In our sample, GRB 021004 has a very bright afterglow, but the host galaxy does not appear to show unusual dust content. On the other hand, GRB 080607 is a dark GRB with large extinction along the line of sight. Their host galaxies have measured $345 \mathrm{GHz}$ fluxes of $0.17 \pm 0.11$ and $0.31 \pm 0.09 \mathrm{mJy}$, respectively. Statistically, we cannot rule out the possibility that the two host galaxies have comparable submillimeter fluxes. Despite that we had already pushed the sensitivities to roughly an or- der of magnitude deeper than previous measurements, deeper ALMA observations (and a larger sample) are clearly needed to tell the difference between the host galaxies of typical and dark GRBs.

On the other hand, the ALMA sensitivity limit is deep enough to probe beyond the ULIRG regime. Chen et al. (2010) suggest that the high-redshift infrared luminous galaxy population contributes to the GRB host galaxy population. The ALMA sensitivity thus allows us to examine whether the two host galaxies are similar to typical dusty galaxies selected by submillimeter telescopes (submillimeter galaxies, hereafter SMGs). First, it is established that bright 345 $\mathrm{GHz}$ selected SMGs primarily reside in the redshift range of $z=1.5-3.5$ (Barger, Cowie, \& Richards 2000; Chapman et al. 2003, 2005; Wardlow et al. 2011). The host galaxies of GRB 021004 and GRB 080607 have redshifts in the range of these bright SMGs. The integrated source counts indicate that bright SMGs of $S_{345}>2$ mJy contribute to $\sim 30 \%$ of the extragalactic background light in this wavelength range (e.g., Coppin et al. 2006). Faint-end counts derived from lensing cluster surveys indicate that approximately $50 \%$ of the background arises from fainter sources in the flux range of $S_{345}=0.5-2$ mJy (Cowie, Barger, \& Kneib 2002; Knudsen, van der Werf, \& Kneib 2008; Chen, Cowie, \& Wang et al. 2011). Our ALMA detection and tight upper limit on the host galaxies of GRB 021004 and GRB 080607 thus put them at the still fainter end of the $345 \mathrm{GHz}$ population.

We further compare the two GRB host galaxies with normal star forming galaxies at high redshift. There exists a correlation between SFR and stellar mass of star forming galaxies at high redshift (e.g., Daddi et al. 2007; Pannella et al. 2009; Karim et al. 2011; Rodighiero et al. 2011). This correlation is often referred to as the star formation "main sequence" of galaxies. Galaxies at the main sequence are suggested to be disks that undergo quasi-steady star formation, and outliers are suggested to be starbursts with star formation boosted by gas-rich mergers (Daddi et al. 2010; Genzel et al. 2010). For $z \sim 2$, Daddi et al. (2007) found SFR $=200 M_{11}^{0.9}\left(M_{\odot} \mathrm{yr}^{-1}\right)$ for main-sequence galaxies, where $M_{11}$ is stellar mass in units of $10^{11} M_{\odot}$. The host galaxy of GRB 021004 has estimated stellar masses of $1.6 \times 10^{10} M_{\odot}$ (Savaglio, Glazebrook, \& Le Borgne 2009) and $2.6 \times 10^{9} M_{\odot}$ (Chen et al. 2009). With our ALMA SFR upper limit $(3 \sigma)$ of $53 M_{\odot} \mathrm{yr}^{-1}$, its SFR/ $M_{11}^{0.9}$ has values of $<280$ or $<1400$, depending on the adopted stellar mass. The former is consistent with main-sequence galaxies, while the latter is close to a starburst. However, both values are 3- $\sigma$ upper limits. The host galaxy of GRB 080607 has a better constrained stellar mass of $1-3 \times 10^{10} M_{\odot}$ (Chen, Perley, \& Wilson et al. 2011). If we adopt our ALMA SFR of 41-77 $M_{\odot} \mathrm{yr}^{-1}$, then it has SFR $/ M_{11}^{0.9}=100-600$. This exercise shows that both GRB host galaxies are consistent with being main-sequence star-forming galaxies.

Finally, the afterglow spectrum of GRB 080607 shows a fairly large dust extinction of $A_{V}=3.2$, and unprecedentedly high gas densities of $N_{\mathrm{HI}}=10^{22.70 \pm 0.15} \mathrm{~cm}^{-2}$ and $N_{\mathrm{CO}}=$ $10^{16.5 \pm 0.3} \mathrm{~cm}^{-2}$, with a warm $\mathrm{CO}$ excitation temperature of $T_{\mathrm{ex}}^{\mathrm{CO}}>100 \mathrm{~K}$ (Prochaska et al. 2009). However, Prochaska et al. also suggest that the intervening molecular cloud is not the birth place of the GRB. The extinction in the afterglow is significantly larger than that for the host galaxy $\left(A_{V}=1.2\right.$, Chen, Perley, \& Wilson et al. 2011). All the above, together with our ALMA result of a relatively normal SFR, indicates that GRB 080607 is not in a rare dusty galaxy, but the sightline happens to pass through a molecular cloud in its host galaxy. 
We thank the referee for the useful comments. This paper makes use of the ALMA data: ADS/JAO.ALMA\#2011.0.00101.S. ALMA is a partnership of ESO (representing its member states), NSF (USA) and NINS (Japan), together with NRC (Canada) and NSC and ASIAA (Taiwan), in cooperation with the Republic of Chile. The JAO is operated by ESO, AUI/NRAO and NAOJ. This work is supported by the NSC grant 99-2112-M-001012-MY3 (W.H.W.).

\section{REFERENCES}

Barger, A. J., Cowie, L. L., \& Richards, E. A. 2000, AJ, 119, 2092

Berger, E., Cowie, L. L., Kulkarni, S. R., Frail, D. A., Aussel, H., \& Barger, A. J. 2003, ApJ, 588, 99

Blain, A. W., \& Longair, M. S. 1993, MNRAS, 264, 509

Blain, A. W., \& Natarajan, P. 2000, MNRAS, 312, 35

Castro-Tirado, A. J., et al. 2010, A\&A, 517, 61

Cenko, S. B., et al. 2009, ApJ, 693, 1484

Chapman, S. C., Blain, A. W., Ivison, R. J., \& Smail, I. R. 2003, Nature, 422, 695

Chapman, S. C., Blain, A. W., Smail, I., \& Ivison, R. J. 2005, ApJ, 622, 772

Chary, R., \& Elbaz, D. 2001, ApJ, 556, 562 (CE01)

Chen, H.-W., et al. 2009, ApJ, 691, 152

Chen, H.-W., et al. 2010, ApJ, 723, L218

Chen, H.-W., Perley, D. A., \& Wilson, C. D., et al. 2011, ApJ, 727, L53

Chen, C.-C., Cowie, L. L., Wang, W.-H., Barger, A. J., \& Williams, J. P. 2011 , ApJ, 733, 64

Christensen, L., Hjorth, J., \& Gorosabel, J. 2004, A\&A, 425, 913

Coppin, K., et al. 2006, MNRAS, 372, 1621

Cowie, L. L., Barger, A. J., \& Kneib, J.-P. 2002, AJ, 123, 2197

Daddi, E., et al. 2007, ApJ, 670, 156

Daddi, E., et al. 2010, ApJ, 714, L118

de Ugarte Postigo, A., et al. 2005, A\&A, 443, 841

Djorgovski, S. G., Frail, D. A., Kulkarni, S. R., Bloom, J. S., Odewahn, S. C. \& Diercks, A. 2001, ApJ, 562, 654

Fynbo, J. P. U., et al. 2005, ApJ, 633, 317

Genzel, R., et al. 2010, MNRAS, 407, 2091

Greiner, J., et al. 2009, ApJ, 693, 1610

Hjorth, J., et al. 2012, ApJ, 756, 187

Jakobsson, P., Hjorth, J., Fynbo, J. P. U., Watson, D., Pedersen, K., Björnsson, G., \& Gorosabel, J. 2004, ApJ, 617, L21

Jakobsson, P., et al. 2005, MNRAS, 362, 245
Karim, A., et al. 2011, ApJ, 730, 61

Kawai, N., et al. 2006, Nature, 440, 184

Kennicutt, R. C., Jr. 1998, ARA\&A, 36, 189

Knudsen, K. K., van der Werf, P. P., \& Kneib, J.-P. 2008, MNRAS, 384, 1611

McMullin, J. P., Waters, B., Schiebel, D., Young, W., \& Golap, K. 2007, Astronomical Data Analysis Software and Systems XVI (ASP Conf. Ser

376), ed. R. A. Shaw, F. Hill, \& D. J. Bell (San Francisco, CA: ASP), 127

Melandri, A., et al. 2008, ApJ, 686, 1209

Melandri, A., et al. 2012, MNRAS, 421, 1265

Michałowski, M. J., et al. 2012, MNRAS, 421, 1265

Pannella, M., et al. 2009, ApJ, 698, L116

Perley, D. A., et al. 2009, AJ, 138, 1690

Perley, D. A., et al. 2011, AJ, 141, 36

Priddey, R. S., Tanvir, N. R., Levan, A. J., Fruchter, A. S., Kouveliotou, C. Smith, I. A., \& Wijers, R. A. M. J. 2006, MNRAS, 369, 1189

Prochaska, J. X., et al. 2009, ApJ, 691, L27

Rodighiero, G., et al. 2011, ApJ, 739, L40

Salvaterra, R., et al. 2009, Nature, 461, 1258

Savaglio, S., Glazebrook, K., \& Le Borgne, D. 2009, ApJ, 691, 182

Sheffer, Y., Prochaska, J. X., Draine, B. T., Perley, D. A., \& Bloom, J. S. 2009, ApJ, 701, L63

Smith, I. A., et al. 2005, A\&A, 439, 987

Tanvir, N. R., et al. 2004, MNRAS, 352, 1073

Tanvir, N. R., et al. 2009, Nature, 461, 1254

Totani, T. 1999, ApJ, 511, 41

Wardlow, J. L., et al. 2011, MNRAS, 415, 1479

Wijers, R. A. M. J., Bloom, J. S., Bagla, J. S., \& Natarajan, P. 1998, MNRAS, 294, 13

Woosley, S. E., \& Heger, A. 2006, ApJ, 637, 914 